\title{
Lung nodule detection on thoracic computed tomography images: Preliminary evaluation of a computer-aided diagnosis system
}

\author{
Metin N. Gurcan, Berkman Sahiner, ${ }^{\text {a) }}$ Nicholas Petrick, Heang-Ping Chan, \\ Ella A. Kazerooni, Philip N. Cascade, and Lubomir Hadjiiski \\ Department of Radiology, University of Michigan, Ann Arbor, Michigan 48109-0904
}

(Received 29 October 2001; accepted for publication 20 August 2002; published 23 October 2002)

\begin{abstract}
We are developing a computer-aided diagnosis (CAD) system for lung nodule detection on thoracic helical computed tomography (CT) images. In the first stage of this CAD system, lung regions are identified by a $k$-means clustering technique. Each lung slice is classified as belonging to the upper, middle, or the lower part of the lung volume. Within each lung region, structures are segmented again using weighted $k$-means clustering. These structures may include true lung nodules and normal structures consisting mainly of blood vessels. Rule-based classifiers are designed to distinguish nodules and normal structures using 2D and 3D features. After rule-based classification, linear discriminant analysis (LDA) is used to further reduce the number of false positive (FP) objects. We performed a preliminary study using $1454 \mathrm{CT}$ slices from 34 patients with 63 lung nodules. When only LDA classification was applied to the segmented objects, the sensitivity was 84\% (53/63) with 5.48 (7961/1454) FP objects per slice. When rule-based classification was used before LDA, the free response receiver operating characteristic (FROC) curve improved over the entire sensitivity and specificity ranges of interest. In particular, the FP rate decreased to 1.74 (2530/1454) objects per slice at the same sensitivity. Thus, compared to FP reduction with LDA alone, the inclusion of rule-based classification lead to an improvement in detection accuracy for the CAD system. These preliminary results demonstrate the feasibility of our approach to lung nodule detection and FP reduction on CT images. (C) 2002 American Association of Physicists in Medicine. [DOI: 10.1118/1.1515762]
\end{abstract}

Key words: computer-aided diagnosis, computed tomography, lung nodule, rule-based classification, linear discriminant analysis

\section{INTRODUCTION}

Lung cancer is the leading cause of cancer deaths in the United States. ${ }^{1}$ The overall 5-year survival rate for lung cancer is $14 \%$. The survival rate increases to $49 \%$ if it is localized, and decreases to about $2 \%$ if it has metastasized. While breast, colon, and prostate cancer have seen improved survival rates between the 1974-1990 time period, there has been no significant improvement in the survival of patients with lung cancer. ${ }^{2}$ This may be due to the lack of a proven screening test. Periodic screening using $\mathrm{CT}$ in prospective cohort studies has been found to improve stage distribution and resectability of lung cancer. ${ }^{3-6}$ Initial findings from a baseline screening of 1000 patients in the Early Lung Cancer Action Project (ELCAP) indicated that low dose CT can detect four times more malignant lung nodules than chest $\mathrm{X}$-ray (CXR), and six times more stage I malignant nodules, which potentially are more treatable. ${ }^{4}$ The number of images that need to be interpreted in CT screening is high, particularly when multi-detector helical CT and thin collimation are used. The analysis of CT images to detect lung nodules is a demanding task for radiologists. Although CT has a much higher sensitivity than CXR, missed cancers are not uncommon in CT interpretation. ${ }^{7-10}$ Double reading is being used in a Japanese CT screening program to reduce missed diagnosis, but it doubles the demand on radiologists' time. It has been demonstrated in mammographic screening that computer-aided diagnosis (CAD) can increase the sensitivity of breast cancer detection in a clinical setting. ${ }^{11}$ CAD may also be a viable alternative to double reading for detection of lung cancer on $\mathrm{CT}$ images.

Because of the potential for CAD to increase the accuracy of lung cancer detection with helical CT, researchers have recently begun to explore CAD methods in this area. In Japan, researchers developed a prototype system and reported high detection sensitivity in an initial evaluation. ${ }^{12,13}$ They used gray-level thresholding to segment the lung region. Next, blood vessels and nodules were segmented using the fuzzy clustering method. The artifacts and small regions were reduced by thresholding and morphological operations. Several features were extracted to differentiate between the blood vessels and nodules. Most of the false positive nodule candidates were reduced through rule-based classification. Lou et al. developed an object-based deformation technique for nodule detection in CT images and initial segmentation on 18 cases was reported. ${ }^{14}$ Fiebich et al. ${ }^{15}$ and Armato et al. ${ }^{16}$ reported the performance of their automated nodule detection schemes on 17 cases. The sensitivity and specificity were $95.7 \%$, with 0.3 false positive (FP) per image in the former study, and $72 \%$ with 4.6 FPs/image in the latter. However, a recent evaluation of the CAD system from the group of Fiebich et al. ${ }^{17}$ on $26 \mathrm{CT}$ exams resulted in a much lower sensitivity of $30 \%$, at 6.3 FPs per CT study. Armato 
et al. ${ }^{18,19}$ recently reported $71 \%$ and $70 \%$ sensitivity, respectively, with 1.5 FPs/image in a data set of 43 cases. They used multilevel gray-level segmentation for the extraction of nodule candidates from CT images. Ko et al. ${ }^{20}$ developed a system that semiautomatically identified nodules, quantified their diameter, and assessed for change in size at follow-up. They reported an $86 \%$ detection rate at $2.3 \mathrm{FPs} / \mathrm{image}$ in 16 $\mathrm{CT}$ studies and found that the assessment of nodule size change by the computer was comparable to that by a thoracic radiologist. Hara et al. used template matching techniques to detect nodules. ${ }^{21}$ The size and the location of the 2D Gaussian templates were determined by the genetic algorithm. The sensitivity of the system was $77 \%$ at a $2.6 \mathrm{FP} / \mathrm{image}$. These reports indicate that computerized detection for lung nodules in helical CT images is promising. However, they also demonstrate large variations in performance, indicating that the computer vision techniques in this area have not been fully developed. Continued effort will be required to bring the performances of these computerized detection systems to a level acceptable for clinical implementation.

We are developing a CAD system for lung cancer detection on helical CT images. The feasibility of our approach was demonstrated in a previous pilot study with 17 cases. ${ }^{22}$ In this study, we further designed knowledge-based segmentation techniques to delineate the lung fields and nodule candidates in different lung regions. False-positive nodules were reduced by rule-based classification in combination with a statistical classifier. The computer vision techniques and the preliminary results for our lung nodule detection system are detailed in this paper.

\section{MATERIALS AND METHODS}

\section{A. Data set}

We analyzed CT studies from 34 patients containing 1454 image slices. The number of $\mathrm{CT}$ slices per patient varied between 14 and 82 with a median of 45 . These cases were acquired from our patient files with approval from our Institutional Review Board. Nineteen of the patients were male and 15 were female. The average age of the patients was 60 . The CT images were acquired using HiSpeed Advantage, CT/i and LightSpeed (GE Medical Systems; Milwaukee, WI) scanners. Tube voltage was $120 \mathrm{kVp}$. Tube current ranged from $180 \mathrm{mAs}$ to $330 \mathrm{mAs}$. Slice thickness was $5 \mathrm{~mm}$, except for two series for which the thickness was $2.5 \mathrm{~mm}$ and $3 \mathrm{~mm}$. Some of the patients had studies with thinner slices. However, these were not used in this study because CAD systems for lung nodules are currently being considered for screening and a thick-slice protocol is generally preferred for screening large populations. For patient files with repeated exams, only the first CT exam at $5 \mathrm{~mm}$ thickness was used. The resolution of the images on the $x-y$ plane varied between $0.546 \mathrm{~mm}$ and $0.761 \mathrm{~mm}$, depending on the physical size of the patient. Each image was $512 \times 512$ pixels and the gray level depth was 12 bits.

A total of 63 lung nodules were marked on the hardcopy films by an experienced chest radiologist. The number of nodules in each case ranged from 1 to 6 . The radiologist-

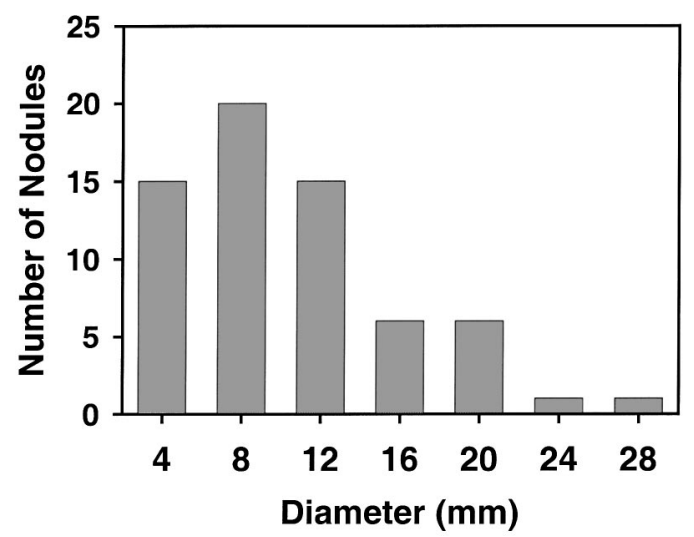

FIG. 1. Distribution of nodule sizes for our data set. The nodule size was measured by a radiologist on hardcopy CT films as the largest diameter of the nodule in any $2 \mathrm{D}$ slice.

identified nodules were used as the gold standard to analyze the accuracy of computerized detection. Eight of the nodules were malignant and 55 were benign, as proven by biopsy or follow-up. The average, standard deviation, minimum and maximum nodule sizes on the $x-y$ plane (slice) were 8.89 $\mathrm{mm}, 5.66 \mathrm{~mm}, 2 \mathrm{~mm}$, and $25 \mathrm{~mm}$, respectively. Figure 1 shows the distribution of nodule sizes in our data set. As can be seen from this histogram, most of the nodules were smaller than $12 \mathrm{~mm}$.

\section{B. Lung region extraction}

The various processing steps in our lung nodule detection system are shown in Fig. 2. The first step is the extraction of the lung regions.

CT lung density varies according to the depth of inspiration, beam collimation, and calibration of the CT scanner. Therefore, we did not use a constant (or a range of) threshold value to segment the lung regions in this study. Instead, an adaptive scheme was implemented. First, a low threshold $(-800 \mathrm{HU})$ value was used to exclude the region external to the thorax. The CT values of the mediastinum and lung walls are much higher than that of the air surrounding the patient. This approximate threshold can successfully separate the sur-

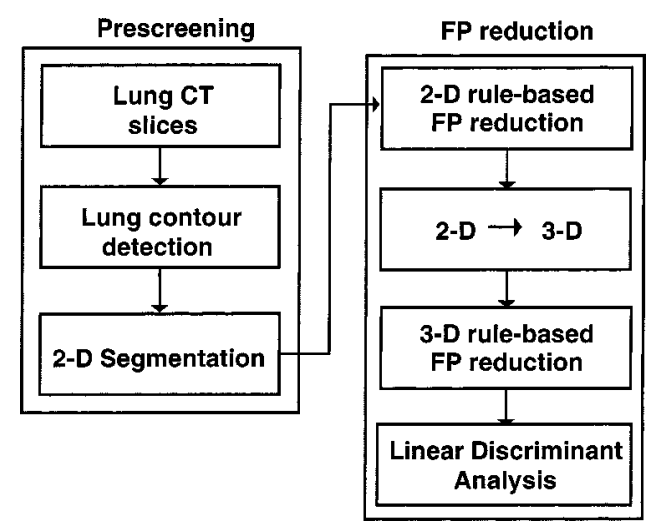

FIG. 2. Schematic illustrating the processing steps in the CT lung nodule detection system. 


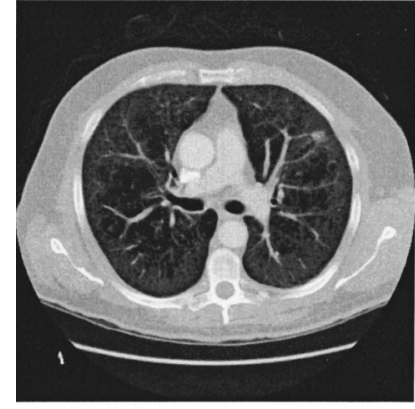

(a)

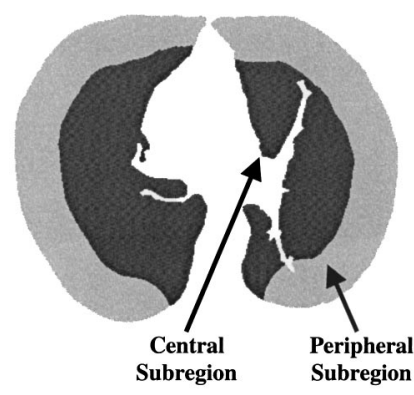

(b)
FIG. 3. (a) Original CT image, and (b) lung region segmentation, and further partitioning of the lung into central and peripheral subregions.

rounding air region and the thorax for all cases. Within the thorax region, the histogram of gray values shows a bimodal distribution. A $k$-means clustering technique ${ }^{23}$ with a ratio threshold of $R=1$ was used to automatically segment the lung regions within the thorax. In clustering, only one feature, the pixel gray level value, was used.

After the lung regions were segmented, left and right lungs were detected. The lung region on each slice was further separated into central and peripheral subregions as shown in Fig. 3. All pixels that are within a 40 pixel distance from the edge of the lung were assigned to the peripheral subregion, and the remaining pixels constituted the central subregion. The central subregion is approximately the hilum. This partitioning was later used in rule-based FP reduction, as explained in Sec. II D.

\section{Lung nodule candidate detection}

Once the lung contours were determined, suspicious structures were segmented within the lung regions by a prescreening program. This program performed weighted $k$-means clustering segmentation with two output classes. The first class included the lung nodule candidates, and the second class was the background within the lung region. The weighted $k$-means clustering algorithm was similar to that we used previously to segment mass from its surrounding tissue on mammograms. ${ }^{23}$ Briefly, in the current application, the image features from both the original image and a median-filtered image were used in the formulation of a feature vector. The median filter had a $5 \times 5$ kernel. The Euclidean distances of the feature vector of each pixel from the two cluster centers were calculated, and the ratio of the two distances (distance to background cluster center/distance to nodule candidate cluster center) was found. If the ratio was larger than a ratio threshold $R$, then the pixel was assigned to the object cluster, otherwise, it was assigned to the background cluster. The cluster centers were updated when new members were included. The procedure was performed iteratively until the cluster centers stabilized. The ratio threshold $R$ controls the relative numbers of the pixels that are classified as background and object pixels. If $R$ is very large, a very small number of pixels are classified as object pixels.
When $R$ is decreased, the ratio of the pixels classified as object pixels increases. The choice of $R$ in this study is described next.

The characteristics of normal structures such as blood vessels depend on their location in the lungs. For example, the vessels in the middle lung region tend to be large and intersect the slices at oblique angles. The vessels in the upper lung regions are usually smaller and tend to intersect the slices more vertically. In general, the vessels are densely distributed near the hilum and spread out towards the periphery. In order to effectively reduce FP objects, different segmentation techniques and classifiers need to be designed based on the characteristics in each region. In this preliminary study, each lung slice was manually classified as belonging to the upper, middle, or lower lung volume by considering whether the mediastinum and the diaphragm were visible in the slice. The ratio threshold $R$ of the $k$-means clustering algorithm was adjusted in the upper, lower, and middle regions of the lung according to the a priori knowledge about the prominence of the normal but bright structures, mainly vessels, in each region. In the upper and lower regions, where the vessels are not very prominent, the value of $R$ was selected as 8.0 after initial experimentation with a small number of cases. In these two regions, since the fraction of pixels that are brighter than the surrounding area is not very large, a relatively large number for $R$ worked satisfactorily. In the middle region, where the vessels are more prominent, the fraction of bright pixels is larger, and a high value for the $R$ may result in missed nodules. For this reason, the ratio threshold was chosen as $R=4.0$ for the middle region. Lung nodule segmentation was performed on each individual slice. The output of the clustering algorithm was a binary image. Due to the segmentation process, some of the segmented binary objects, which represented nodule candidates, contained holes. The holes within the $2 \mathrm{D}$ binary images were filled using a flood-filled algorithm ${ }^{24}$ because nodule candidates were treated as solid objects.

\section{Detection of juxta-pleural nodule candidates}

Some of the nodules adjacent to the pleura (juxta-pleural nodules) may be excluded from the extracted lung regions as shown in Figure 4. In our data set, 5 of the 63 nodules were juxta-pleural nodules. Armato et al. ${ }^{18,19}$ used a rolling ball algorithm to recover these nodules. A two-dimensional circle (rolling ball) was moved around the lung contour. When the circle touched the contour at more than one point, these points were connected by a line. In the studies by Kanazawa et $a l .{ }^{12}$ and Ko et al., ${ }^{20}$ the curvatures of the lung border were calculated and the border was corrected at locations of rapid curvature change by straight lines. In our application, a novel technique, referred to as the indentation detection method, was designed to detect juxta-pleural nodules. First, a closed contour along the boundary of the lung was constructed using a boundary tracking algorithm. For every pair of points $P_{1}$ and $P_{2}$ along this contour, three distances were calculated. The first two distances, $d_{1}$ and $d_{2}$, were the distances between $P_{1}$ and $P_{2}$ measured by traveling along the 


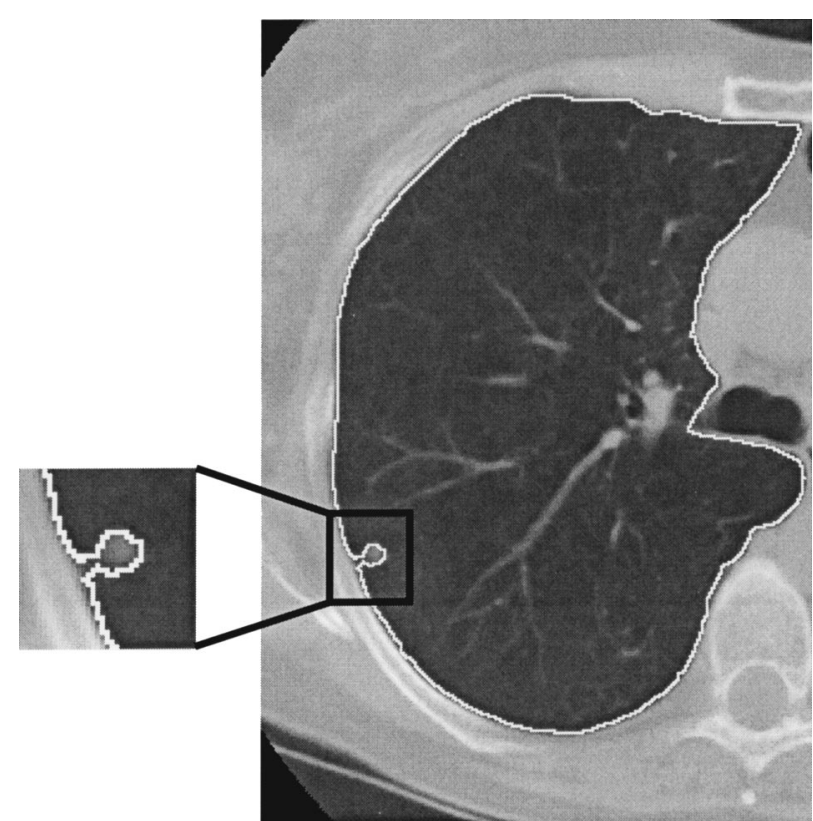

FIG. 4. A lung nodule adjacent to pleura. The segmented lung region marked by the white border excludes the lung nodule.

contour in the counter-clockwise and clockwise directions, respectively. The third distance, $d_{e}$, was the Euclidean distance, which is the length of the line connecting $P_{1}$ and $P_{2}$. Next, the ratio of the minimum of the first two distances to the Euclidean distance was calculated

$$
R_{e}=\frac{\min \left(d_{1}, d_{2}\right)}{d_{e}} .
$$

If this ratio, $R_{e}$, was greater than a preselected threshold, the lung contour between $P_{1}$ and $P_{2}$ was corrected using a straight line through $P_{1}$ and $P_{2}$. The value for this threshold was chosen as 1.5 in this study.

\section{E. Rule-based FP reduction}

Figure 5 shows the segmented structures for a sample CT slice. In this slice, the true lung nodule was segmented along with normal lung structures (mainly blood vessels) with high intensity values. In most cases, these normal lung structures could be reduced based on their morphology. Therefore, we designed rule-based classifiers to distinguish nodules and blood vessel structures. The first set of two rules was applied to image features extracted from the individual 2D CT slices. The first rule was intended to distinguish thin and long objects, such as vessels, from lung nodules. Each segmented object was fitted by an ellipse and the ratio of the major (a) to minor (b) axis, $R_{t l}$, was calculated as [Fig. 6(a)]

$$
R_{t l}=\frac{a}{b} .
$$

Whenever this ratio, $R_{t l}$, exceeded a threshold, the segmented object was considered to be a blood vessel and eliminated from further processing. This threshold was experimentally determined as 3.0.

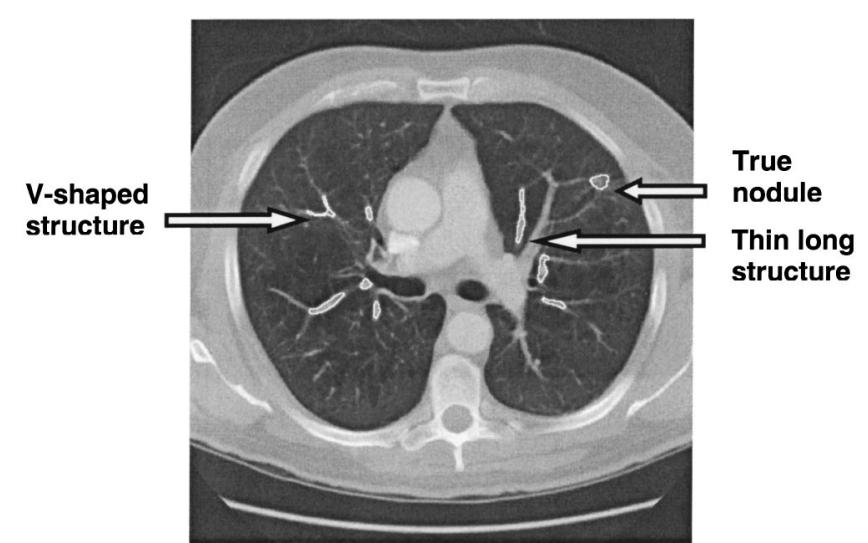

FIG. 5. Output of the prescreening program. Borders of the detected objects are marked. A true nodule as well as other normal structures such as blood vessels are segmented.

Many of the segmented vessels had branching $V$ shapes. Our second rule was a rectangularity criterion (the area of the segmented object to its bounding box) designed to eliminate the $V$-shaped vessels [Fig. 6(b)]

$$
R_{v}=\frac{\text { Area of object }}{\text { Area of bounding box }} .
$$

Whenever the ratio, $R_{v}$, was lower than a threshold, the object was considered to be a blood vessel and it was eliminated from further processing. This threshold was experimentally determined as 2.0.

The remaining 2D segmented objects were connected across consecutive CT slices based on a 26-connectivity rule to form three-dimensional (3D) objects. In 26-connectivity, a voxel $B$ is connected to a voxel $A$ if voxel $B$ is any one of the 26 neighboring voxels on a $3 \times 3 \times 3$ cube centered at voxel A. ${ }^{22}$

FPs were further reduced using four classification rules regarding the size of the bounding box, the maximum object circularity, and the relation of the location of the object to its size. These classification rules were designed by using knowledge of the lung structures and nodules, with additional adjustment of the parameters by experimentation. These rule-based classifiers, like many other classifiers and decision thresholds used in CAD systems, would trade-off a small fraction of sensitivity for the reduction of a large number of FPs. The first two classification rules dictated that the $x$ and $y$ dimensions of the bounding box enclosing the segmented $3 \mathrm{D}$ object had to be larger than 3 pixels. This is

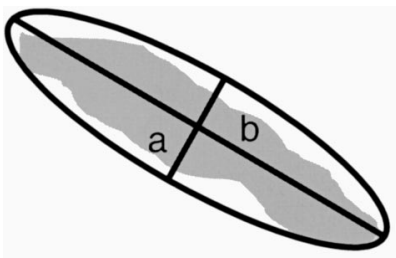

(a)

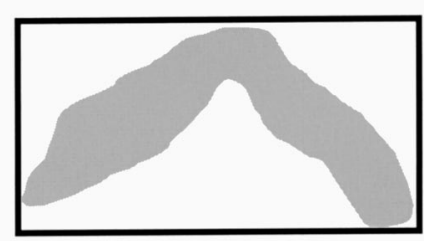

(b)
FIG. 6. Examples of segmented vessel structures (a) thin long, (b) V shaped. 
equivalent to approximately $2 \mathrm{~mm}$ in each dimension (3 $\times 0.581=1.7 \mathrm{~mm}$ for the highest resolution and $3 \times 0.786$ $=2.4 \mathrm{~mm}$ for the lowest resolution). This rule was introduced to remove very small nodule candidates, which are considered negative in many screening protocols. The third classification rule was based on circularity because true nodules are expected to exhibit some circularity. The rule required that the maximum circularity of the cross sections of the segmented 3D object among the slices containing the object be greater than 0.2 . This very lax circularity threshold reduced the number of FP structures while not affecting the detection of any of the true nodules. The fourth rule was based on the knowledge that $80-90 \%$ of the nodules are peripherally located within the subpleural regions and outer one-third of the lungs. ${ }^{25}$ In a single cohort screening study of 1359 patients in Japan by Kaneko et al., all 15 cancers found were described as peripheral. ${ }^{6}$ The fourth rule eliminated lung nodule candidates in the central lung region that were smaller than $3 \mathrm{~mm}$ in the longest dimension. The four rules were applied after forming 3D objects, therefore small objects that were part of a nodule would not be eliminated.

\section{F. Feature extraction}

Once 3D objects were formed, the following features were extracted for each 3D object: volume, surface area; average gray value; standard deviation, skewness and kurtosis of the gray value histogram. The volume was calculated by counting the number of voxels within the object and multiplying this by the unit volume of a voxel. For surface area calculation, the voxels along the surface of the object were considered. The top and bottom voxels contributed to the top and bottom area calculation and the other peripheral voxels contributed to the side surface calculation. Due to anisotropy of CT imaging, areas of these regions should be calculated separately and then combined to obtain the total surface area. In addition, area, perimeter, circularity, compactness; major and minor axes and their ratio, and eccentricity of a fitted ellipse in each cross section of the 3D object were also calculated. The maximums of these values were used as the objects's 2D feature values. While calculating 2D features, the bit quads algorithm was employed to calculate the total area and total perimeter of the $2 \mathrm{D}$ objects in a fast manner. ${ }^{24}$

\section{G. FP reduction using an LDA classifier}

After rule-based classification, a linear discriminant analysis (LDA) classifier was used to further reduce the number of FP objects. ${ }^{26} \mathrm{LDA}$ is a statistical method to determine the dividing boundaries that separate two or more groups by using a number of features. It can yield the optimal boundaries on the basis of combination of features. Several widely available statistical packages can be used to perform LDA. Using a stepwise feature selection process, ${ }^{27}$ the most discriminating features were determined to be the volume, surface area, average and standard deviation of gray values and the maximum values of the cross sectional area, perimeter, circularity, major and minor axes, and eccentricity.

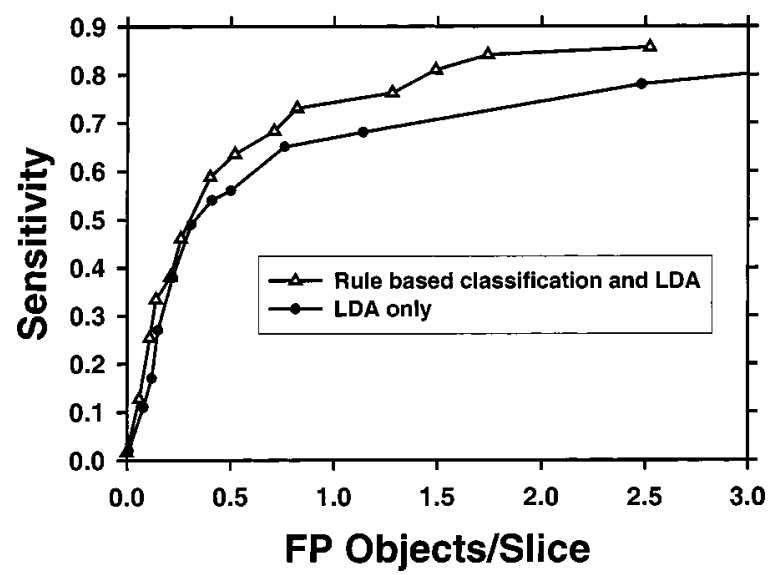

FIG. 7. The FROC curves of the combined FP reduction technique and only the LDA.

These features were used as predictor variables in the LDA classifier, which was designed using a leave-one-case-out training and test resampling scheme.

\section{RESULTS}

We have performed a preliminary study to evaluate the feasibility of our approach for detection and differentiation of true nodules and FP structures on thoracic helical CT images. The prescreening stage detected $90 \%(57 / 63)$ of the nodules with an average of $12.92 \mathrm{D}$ objects per slice. The missed nodules were all less than $5 \mathrm{~mm}$ and benign. After the first rule-based classification stage, there were a total of 8455 3D objects, including $90 \%$ of the true nodules.

After FP reduction using the rules based on the bounding box size and circularity, and reduction of small nodule candidates in the central lung region, there were a total of 4247 nodule candidates at a sensitivity of $87 \%$ (55/63). The LDA classifier reduced the number of FP objects to 2527, corresponding to $1.74(2527 / 1454)$ FP objects per slice, at a detection sensitivity of $84 \%(53 / 63)$. The free response receiver operating characteristic (FROC) ${ }^{28}$ curve of this combined FP reduction technique is shown in Fig. 7. This curve was obtained by varying the detection threshold of the LDA scores and calculating the number of FPs and the sensitivity level corresponding to that threshold. To demonstrate the advantage of rule-based classification, the lower FROC curve shows the detection results if only the LDA classifier was used. When LDA classification alone was applied to the segmented objects, the sensitivity was $84 \%$ (53/63) with 5.48 (7961/1454) FP objects per slice.

\section{DISCUSSION}

Rule-based systems usually lack the generalizability property and therefore should be carefully used in computer vision applications. In this work, we designed rules to reduce the number of FPs based on knowledge gained from radiologists such as the anatomical characteristics and morphological features of lung nodules (size, location and shape). We attempted to use relatively lax criteria so that they are not too 
specific to the data set used. For instance, we know that lung nodules should exhibit some circularity. By imposing a very low circularity threshold (0.2), we reduce the number of FP structures while not affecting the detection of any of the true nodules. However, since the data set was small in this preliminary study, further work with a larger data set will be required to evaluate the robustness of the rules.

In the current application, the segmentation was carried out in 2D while 3D information was included after the segmentation. True 3D segmentation may provide more accurate shapes of the 3D structures. However, it is common to encounter a "leaking" problem (i.e., different objects are combined into a single set of contiguous voxels ${ }^{29}$ ) in 3D segmentation. Our 2D vessel reduction rules (Sec. II D) partially solved this problem by first eliminating a large number of vessel structures during the $2 \mathrm{D}$ analysis before forming $3 \mathrm{D}$ objects.

While calculating the features for FP reduction in the LDA classification step, 2D features were calculated from each slice of the objects. It is not appropriate to use all of these $2 \mathrm{D}$ features because not all object slices provide useful information. For example, the top and the bottom slices of an object might be very small. Features based on these small 2D structures may not represent the general characteristics of a nodule. Therefore, the maximum of each 2D feature among all slices of the 3D object was employed in the feature space for FP reduction. Future extension of the 3D features will include 3D shape descriptors such as sphericity, smoothness of the 3D object surface, branching and connectivity in 3D. These features may provide a better 3D perspective of the objects and thus improve differentiation of nodules and normal pulmonary structures.

In this feasibility study, our lung nodule detection algorithm has not been fully automated. The classification of the lungs into the upper, middle, and lower regions was manually performed. This step can be automated by use of knowledge-based classification rules. ${ }^{29}$ More specific feature extraction and classification criteria tailored to the different regions will also need to be designed to better exploit the different characteristics of blood vessels and normal structures in these regions for FP reduction. Fuzzy classification rules in place of our current rule-based classification may better accommodate the large variations in the features of the nodules and normal structures, and may lead to further improvement in the recognition of FPs. Texture in the tissue regions surrounding the nodule candidates will also be analyzed to differentiate potential differences between growing nodules and normal vessels. However, the design of more complex classification rules and classifiers will have to be based on a larger data set in order to reduce the chance of overtraining. Further studies are underway to pursue these issues for our lung nodule detection system.

\section{CONCLUSIONS}

In this work, the feasibility of our approach to lung nodule detection using rule-based and statistical classifiers in CT images is demonstrated. A statistical classifier combined with a rule-based classifier achieved $84 \%$ sensitivity at $1.74 \mathrm{FP} /$ section. Although the number of FPs is still large, the performance is comparable to those reported by other groups. ${ }^{19-21}$

In this study, we improved the lung nodule detection system by using knowledge gained from radiologists such as anatomical characteristics and morphological features of lung nodules (size, location, and shape) and the characteristics of normal structures such as blood vessels in different regions of the lungs. We designed rule-based classifiers to incorporate this knowledge into our CAD system, and chose decision rules based on the anatomical locations. Our results indicate that combining rule-based classification with a statistical classifier can reduce the number of FPs without a significant impact on the sensitivity of lung nodule detection, in comparison to using a statistical classifier alone, thereby leading to an overall improvement in the FROC performance and the detection accuracy of the CAD system.

One of the concerns of using CT for lung cancer screening is the large number of images that need to be evaluated. The increased workload on interpretation of CT studies can be a potential source of fatigue and error in detecting subtle lesions. When fully developed, a CAD system can bring several benefits to lung cancer screening. A CAD system may improve the sensitivity of cancer detection, reduce oversight errors, and decrease inter- and intra-reader variations.

\section{ACKNOWLEDGMENTS}

This work is supported by a USPHS Grant No. CA 93517 and a Career Development Award (B.S.) from the USAMRMC (DAMD 17-96-1-6012). The content of this publication does not necessarily reflect the position of the government and no official endorsement of any equipment and product of any companies mentioned in the publication should be inferred. The authors are grateful to Charles E. Metz, Ph.D., for providing the LABROC program.

\footnotetext{
${ }^{a}$ Electronic mail: berki@umich.edu

${ }^{1}$ American Cancer Society, "American Cancer Society-Cancer Facts and Figures 2001," www.cancer.org (2001).

${ }^{2}$ National Cancer Institute, "Surveillance, Epidemiology, and End Results (SEER) Program Public-Use CD-ROM (1973-1997)," DCCPS, Cancer Surveillance Research Program, Cancer Statistics Branch, 2000.

${ }^{3}$ G. M. Strauss, R. E. Gleason, and D. J. Sugarbaker, "Screening for lung cancer: Another look; A different view," Chest 111, 754-768 (1997).

${ }^{4}$ C. I. Henschke et al., "Early lung cancer action project: overall design and findings from baseline screening," Lancet 354, 99-105 (1999).

${ }^{5}$ S. Sone et al., "Mass screening for lung cancer with mobile spiral computed tomography scanner," Lancet 352, 1242-1245 (1998).

${ }^{6}$ M. Kaneko, K. Eguchi, H. Ohmatsu, R. Kakinuma, T. Naruke, K. Suemasu, and N. Moriyama, "Peripheral lung cancer: screening and detection with low-dose spiral CT versus radiography," Radiology 201, 798802 (1996).

${ }^{7}$ C. S. White, B. M. Romney, A. C. Mason, J. H. M. Austin, B. H. Miller, and Z. Protopapas, "Primary carcinoma of the lung overlooked at CT: analysis of fings in 14 patients," Radiology 199, 109-115 (1996).

${ }^{8} \mathrm{~J}$. W. Gurney, "Missed lung cancer at CT: imaging findings in nine patients," Radiology 199, 117-122 (1996).

${ }^{9}$ S. D. Davis, "Through the "retrospectoscope": a glimpse of missed lung cancer at CT," Radiology 199, 23-24 (1996).

${ }^{10}$ R. Kakinuma, H. Ohmatsu, M. Kaneko, K. Eguchi, T. Naruke, K. Nagai, Y. Nishiwaki, A. Suzuki, and N. Moriyama, "Detection failures in spiral
} 
CT screening for lung cancer: analysis of CT findings," Radiology $\mathbf{2 1 2}$, 61-66 (1999).

${ }^{11}$ T. W. Freer and M. J. Ulissey, "Screening mammography with computeraided detection: Prospective study of 12,860 patients in a community breast center," Radiology 220, 781-786 (2001).

${ }^{12}$ K. Kanazawa, Y. Kawata, N. Niki, H. Satoh, H. Ohmatsu, R. Kakinuma, M. Kaneko, N. Moriyama, and K. Eguchi, "Computer-aided diagnosis for pulmonary nodules based on helical CT images," Comput. Med. Imag. Graph., 157-167 (1998).

${ }^{13}$ H. Satoh, Y. Ukai, N. Niki, K. Eguchi, K. Mori, H. Ohmatsu, R. Kakinuma, M. Kaneko, and M. Moriyama, "Computer aided diagnosis system for lung cancer based on retrospective helical CT image," Proc. SPIE 3661, 1324-1335 (1999).

${ }^{14}$ S. L. Lou, C. L. Chang, K. P. Lin, and T. S. Chen, "Object-based deformation technique for 3-D CT lung nodule detection," Proc. SPIE 3661, 1544-1552 (1999).

${ }^{15}$ M. Fiebich, C. Wietholt, B. C. Renger, S. G. Armato, K. R. Hoffmann, and S. Dietrich, "Automatic detection of pulmonary nodules in low-dose screening thoracic CT examinations," Proc. SPIE 3661, 1434-1439 (1999).

${ }^{16}$ S. G. Armato, M. L. Giger, J. T. Blackburn, K. Doi, and H. MacMahon, "Three-dimensional approach to lung nodule detection in helical CT," Proc. SPIE 3662, 553-559 (1999).

${ }^{17}$ D. Wormanns, M. Fiebich, C. Wietholt, S. Diederich, and W. Heindel, "Automatic detection of pulmonary nodules at spiral CT-first clinical experience with a computer-aided diagnosis system," Proc. SPIE 3979, 129-135 (2000).

${ }^{18}$ S. G. Armato, M. L. Giger, K. Doi, and H. MacMahon, "Computerized lung nodule detection: Comparison of performance for low-dose and standard-dose helical CT scans," Proc. SPIE 4322, 1449-1454 (2001).

${ }^{19}$ S. G. Armato, M. L. Giger, and H. MacMahon, "Automated detection of lung nodules in CT scans: Preliminary results," Med. Phys. 28, 15521561 (2001).
${ }^{20}$ J. P. Ko and M. Betke, "Chest CT: Automated nodule detection and assessment of change over time-preliminary experience," Radiology 2001, 267-273 (2001).

${ }^{21}$ T. Hara, H. Fujita, Y. Lee, H. Yoshimura, and S. Kido, Automated lesion detection methods for 2D and 3D chest x-ray images, 27-29 September 1999.

${ }^{22}$ M. Gurcan, N. Petrick, B. Sahiner, H. P. Chan, P. N. Cascade, E. Kazerooni, and L. M. Hadjiiski, "Computerized lung nodule detection on thoracic CT images: combined rule-based and statistical classifier for false positive reduction," Proc. SPIE 4322, 686-692 (2001).

${ }^{23}$ B. Sahiner, H. P. Chan, N. Petrick, D. Wei, M. A. Helvie, D. D. Alder, and M. M. Goodsitt, "Image feature selection by a genetic algorithm: Application to classification of mass and normal breast tissue on mammograms," Med. Phys. 23, 1671-1684 (1996).

${ }^{24}$ W. K. Pratt, Digital Imaging Processing, 2nd ed. (Wiley, New York, 1991).

${ }^{25}$ S. G. Erberich, K. S. Song, H. Arakawa, H. K. Huang, R. Webb, K. S. Hoo, and B. W. Loo, "Knowledge-based lung nodule detection from helical CT," Radiology 205, 617 (1997).

${ }^{26}$ R. O. Duda and P. E. Hart, Pattern Classification and Scene Analysis (Wiley, New York, 1973)

${ }^{27}$ H. P. Chan, D. Wei, M. A. Helvie, B. Sahiner, D. D. Adler, M. M. Goodsitt, and N. Petrick, "Computer-aided classification of mammographic masses and normal tissue: Linear discriminant analysis in texture feature space," Phys. Med. Biol. 40, 857-876 (1995).

${ }^{28}$ P. C. Bunch, J. F. Hamilton, G. K. Sanderson, and A. H. Simmons, "A free response approach to the measurement and characterization of radiographic observer performance," J. Appl. Photogr. Eng. 4, 166-171 (1978).

${ }^{29}$ B. S. Brown, M. F. McNitt-Gray, N. J. Mankovich, J. G. Goldin, J. Hiller, L. S. Wilson, and D. R. Aberle, "Method for segmenting chest CT image data using an anatomical model: Preliminary results," IEEE Trans. Med. Imaging 16, 828-839 (1997). 